\title{
Las ventajas y desventajas que el vínculo multiempresa y la red de cooperación empresarial internacional presentan para la PYME ${ }^{1}$
}

\author{
Tania Elena González Alvarado - Universidad Nacional Autónoma de México²
}

\begin{abstract}
Resumen
El objetivo del presente trabajo es exponer las ventajas y desventajas que el vínculo multiempresa y la red de cooperación empresarial internacional presentan a la PYME. El vínculo multiempresa permite que la empresa participe en mercados extranjeros al insertarse en una red internacional y, a su vez, propicie el desarrollo local. Para lograr dicho objetivo se analizan cinco casos resaltando en cada uno de ellos las ventajas y desventajas que el vínculo multiempresa ofrece y la forma en que lo aprovechan las empresas involucradas.
\end{abstract}

Palabras clave: vínculo multiempresa, cooperación, acuerdos, PYME.

\section{Abstract}

The aim of this article is to show the advantages and disadvantages that multienterprise tie and networks of enterprise cooperation offer to small and mediumsized enterprises (SMEs). Multi-enterprise tie may contribute to SMEs' participate in international markets and to contribute to local development. In this article five enterprise cases are analyzed. Each case demonstrated the advantage and disadvantages of multi-enterprise tie and the way that SMEs took advantage of them.

Key words: multi-enterprise tie, co-operation, agreements, SMEs

Por lo general, el estudio sobre los vínculos entre empresas se basa en el Análisis de Redes Sociales (Casanueva, 2003). Mediante este enfoque se han creado modelos cuantitativos. Modelos que, si bien han arrojado evidencia importante en el estudio de los vínculos empresariales, también es cierto que al ser cuantitativos no permiten estudiar aspectos de carácter cualitativo que resultan importantes para el avance teórico. Aspectos cualitativos, tales como: bajo qué estrategia se vincula el empresario (de cooperación o de competencia), qué efecto tiene la experiencia de un fracaso en el establecimiento de un nuevo vínculo, cómo permite un vínculo multiempresa que el empresario aprenda a cooperar y cómo este aprendizaje favorece que el empresario establezca vínculos con el extranjero. El enfoque

\footnotetext{
${ }^{1}$ Derivado del proyecto “Las redes de cooperación empresarial internacionales. Creación de valor para la PYME mexicana" inscrito al Programa de Apoyo para los Proyectos de Investigación e Innovación Tecnológica, Dirección General de Apoyo al Personal Académica, Universidad Nacional Autónoma de México.

2 Enviar correspondencia a: Tania Elena González Alvarado. Correo electrónico: tgonza@correo.fca.unam.mx, cub. 24, DICAI, FCA, UNAM, Ciudad Universitaria, Circuito exterior s/n, México, D.F. 04150
} 
REDES- Revista hispana para el análisis de redes sociales

Vol. 14,\#10, Junio 2008

http: //revista-redes.rediris.es

cualitativo enriquece al cuantitativo antes que contraponerse, particularmente en el estudio de las empresas (heterogéneas multidemnsionalmente).

En los modelos cuantitativos se ha mencionado el estudio de los vínculos conforme a sus atributos, tales como: débiles, fuertes, diádicos, triádicos y complejos (Granovetter, 1973; Wasserman y Faust, 1994; Contractor et al., 2006). Poco es lo que se ha estudiado sobre un solo vínculo formado por tres o más elementos que, mediante la cooperación como mecanismo, logren que la centralidad (Freeman, 1978) sea prácticamente nula entre ellos. Creemos que para estudiar esto último es indispensable hacerlo desde un enfoque cualitativo (Yin, 2003).

Por otra parte, la mayoría de los estudios sobre vinculaciones entre empresas se ha centrado en los clusters, distritos industriales $\mathrm{y} / \mathrm{o}$ conglomerados. En otras palabras, se ha basado en el estudio de empresas que se encuentran geográficamente concentradas. Dicha concentración se debe a fuerzas centrípetas (Krugman, 1996) que les permiten permanecer juntas a pesar de que entre ellas imperan las estrategias de competencia (González, 2005), siendo entonces poco representativas para el estudio de los vínculos de cooperación.

En el momento en que se ha abordado la vinculación de empresas en el nivel internacional, la mayor parte de los trabajos se han centrado en el estudio de las trasnacionales y su relación asimétrica con sus sucursales, sus clientes o proveedores de otras regiones.

Hemos pretendido dejar estos tres caminos de lado y tomar uno más adecuado con el fenómeno de estudio. Centramos la atención en empresas de menor dimensión que se encuentran geográficamente dispersas y cuyos empresarios están aprendiendo a establecer vínculos de cooperación en un ambiente internacional. Todo esto desde un enfoque cualitativo en el que el análisis de casos, las entrevistas, la observación y la revisión documental son nuestros mejores aliados para la recuperación de evidencia empírica.

El conocimiento que se puede extraer del estudio cualitativo de dicha vinculación permite explicar cómo una empresa de menor tamaño logra participar en mercados internacionales $\mathrm{y}$, en algunas ocasiones, permite que en una pequeña localidad aumente el nivel de vida de la población. 
REDES- Revista hispana para el análisis de redes sociales

Vol.14,\#10, Junio 2008

http: //revista-redes.rediris.es

El presente trabajo se deriva de un proyecto de investigación en el que se analiza como fenómeno las redes de cooperación empresarial internacionales, usando como herramienta el estudio descriptivo de casos, por tanto, no existe aplicación de modelos matemáticos y/o estadísticos.

Para exponer las ventajas y desventajas ${ }^{3}$ del vínculo multiempresa y la red de cooperación empresarial internacional hemos dividido el trabajo en tres secciones. En la primera se explica brevemente qué es un vínculo multiempresa, cómo interviene el mecanismo de cooperación en su formación y de qué forma facilita la inserción de las empresas en la red de cooperación empresarial internacional, articulando con esto último la red local a una internacional. En la segunda parte se analizan cinco casos sobre el vínculo multiempresa. La tercera sección del trabajo recupera los cinco casos, identificando similitudes y diferencias, para con ello señalar las ventajas y desventajas del vínculo multiempresa y la red de cooperación empresarial internacional. A continuación pasamos a identificar qué es un vínculo multiempresa, cómo se establece teniendo como mecanismo la cooperación y de qué forma es un articulador de la red local a la internacional.

\section{EI vínculo multiempresa, la cooperación como mecanismo y la articulación a la red de cooperación empresarial}

La mayoría de las investigaciones sobre vínculos empresariales y la formación de redes se concentran en los clusters o distritos industriales (Schmitz 1999, De Martino et al. 2006, Grabher e Ibert, 2005; Waxell y Malmberg 2007). Además, estos estudios se basan en modelos cuantitativos que difícilmente permiten detectar, aislar y estudiar los vínculos creados bajo el mecanismo de cooperación. El trabajo de investigación cualitativo que actualmente estamos desarrollando versa sobre el vínculo formado por múltiples empresas, cuyo mecanismo es la cooperación, y que conduce a la formación de redes internacionales. En otras palabras, estudiamos los vínculos con elementos dispersos geográficamente que, difícilmente, entran en el esquema de cluster o distrito industrial. El hecho de no centrarnos sólo en los vínculos locales se debe a que en estos dos últimos

\footnotetext{
3 Esta ventaja se convierte en ventaja competitiva en el momento en que el empresario toma provecho de la situación para beneficio de su empresa. Si analizamos el fenómeno desde la perspectiva del vínculo o la red, como es el caso del artículo, usamos el término ventaja al referirnos a dichas situaciones. Sin embargo, tenga en cuenta el lector que este mismo análisis, si se efectuara desde la perspectiva del empresario y la forma como internamente transforma a la empresa las ventajas ofrecidas por el vínculo y al red, usaríamos el término ventaja competitiva. En todo caso y para esto último tendríamos dos enfoques básicos: en relación con la industria a la que la empresa pertenece (Porter, 1998) y con base en los recursos y capacidades de la empresa (Lippman y Rumelt, 1982; Rumelt, 1984; Barney, 1991). Consideramos que este último enfoque, sobre recursos y capacidades, es fundamental para el análisis del empresario ante la disyuntiva entre cooperar o competir.
} 
REDES- Revista hispana para el análisis de redes sociales

Vol. 14,\#10, Junio 2008

http: // revista-redes.rediris.es

esquemas las empresas, ante la cercanía geográfica, encuentran otras fuerzas centrípetas (Krugman, 1996) para vincularse, siendo poco atractiva la cooperación empresarial. En otras palabras, las empresas que forman parte de estos entramados locales se caracterizan por establecer vínculos bajo estrategias de competencia, antes que de cooperación (González, 2005).

En el estudio de los vínculos empresariales se ha señalado que las empresas establecen relaciones bajo dos estrategias (Lax y Sebenius, 1991): cooperar crear valor para compartirlo- o competir - demandar valor y arrebatarlo. Ambos estrategias ofrecen diversas ventajas y desventajas, las cuales los empresarios evalúan conforme a cada circunstancia para con ello decidir qué camino van a seguir.

Cuando ambos empresarios se proponen crear valor para compartirlo se genera un vínculo bajo el mecanismo de cooperación. Este tipo de vínculo es el que facilita el establecimiento de relaciones entre empresas que pertenecen a distintas regiones del mundo y en el que, por tanto, los empresarios cuentan con distinto idioma y cultura (González 2003).

En muchos estudios se da por sentada la cooperación, se considera que todo vínculo es de cooperación, que todos los acuerdos se establecen bajo el mecanismo de cooperación y no siempre se considera que la empresa puede colaborar bajo el mecanismo de competencia y vincularse con el mecanismo de cooperación ausente. En otras palabras, se presupone que en todo vínculo la empresa coopera; sin embargo, la teoría de juegos (Pérez et al. 2004) nos plantea cuándo se establece un vínculo de competencia en lugar de uno de cooperación. Esto ocurre cuando una o ambas partes utilizan estrategias de competencia. También es importante considerar lo siguiente: con respecto a los acuerdo, por ser incompletos darán paso a la conducta oportunista, por tanto no son garantía de que exista un vínculo de cooperación. Además, la conducta oportunista aumentará entre mayor tiempo abarque el acuerdo (Williamson y Winter, 1996). Un acuerdo por sí mismo no garantiza que el vínculo se mantenga bajo el mecanismo de cooperación. 
REDES- Revista hispana para el análisis de redes sociales

Vol.14,\#10, Junio 2008

http: // revista-redes.rediris.es

En sentido contrario, los vínculos de cooperación empresarial sólo se crearán en la medida en que se elimine la conducta egoísta y se fomente la confianza (Casanueva et al., 2006). Para que un vínculo de cooperación se de, todas las partes involucradas trabajan bajo el mecanismo de crear valor ${ }^{4}$ y compartirlo.

Debido a la incertidumbre ante el futuro, el empresario se enfrentará ante la disyuntiva de cooperar o competir, siendo entonces los vínculos locales los que le brinden la posibilidad de aprender a cooperar y a acumular experiencia sobre la solución de conflictos.

Un vínculo de competencia puede transformarse en un vínculo de cooperación, en la medida en que los implicados aprenden de los fracasos y acumulan experiencia sobre las vinculaciones, sobre las ventajas y desventajas de las mismas. Este paso del mecanismo de competencia al de cooperación puede darse en sentido inverso, cuando la confianza entre las partes se mina y por circunstancias ajenas a su propia voluntad deben permanecer colaborando.

El vínculo se forma por dos empresas (vínculo simple) o excede en número (vínculo múltiple o multiempresa). El vínculo multiempresa es aquel en el que más de dos empresas se vinculan bajo un mismo objetivo. El vínculo multiempresa creado bajo el mecanismo de cooperación difícilmente excederá en número de empresas que lo conforman porque será cada vez más difícil concertar un objetivo común. Por otra parte, El vínculo simple tenderá a convertirse en múltiple, en la medida que el empresario encuentre que, por la racionalidad de grupo, el beneficio obtenido por la cooperación de un número determinado de empresarios es inferior al que se obtendría incorporando un nuevo elemento (Fernández y Arranz 1999). De tal forma que el vínculo simple tenderá a transformarse en múltiple, y el múltiple, a limitar el número de empresas que participan en él. Este límite en el número de empresas que lo conforman existirá sólo cuando se trate de vínculos bajo el mecanismo de cooperación (González, 2004). En este sentido, un vínculo creado bajo el mecanismo de cooperación bien pudiera transformarse, operando bajo el mecanismo de competencia. Sin embargo, el aumento en participantes no es determinante para que este cambio se de, como se verá más adelante al analizar los casos, existen otros factores cualitativos que pueden influir en dicho cambio.

\footnotetext{
4 En el sentido de permitir el acceso a recursos que difícilmente obtendría la empresa por sí sola. Recursos que pueden ser usados a través de las relaciones que los empresarios establezcan. (Casanueva, 2006)
} 
REDES- Revista hispana para el análisis de redes sociales

Vol.14,\#10, Junio 2008

http: // revista-redes.rediris.es

En un vínculo bajo el mecanismo de cooperación se disminuye la centralidad (control) entre los empresarios que conforman el vínculo multiempresa; a su vez, el vínculo multiempresa, como tal, cuenta con una mayor centralidad en relación con las otras empresas que se encuentran fuera del vínculo pero que mantienen relaciones con las empresas que sí forman parte del mismo. Este vínculo multiempresa articula la red local a la internacional. En otras palabras, existe la descentralización en la toma de decisiones dentro del vínculo multiempresa (Sarason, et al. 1978), lo que provoca una mayor sinergia que se observa en el control que este vínculo ejerce hacia fuera del mismo.

Nos referimos a un vínculo múltiple o multiempresa cuando: una empresa establece un vínculo con más de dos empresas; es decir, no ha establecido varios vínculos con más de dos empresas, más bien, todas estas empresas han establecido un solo vínculo bajo el mecanismo de cooperación. Esto último parece difícil de lograr, sino es que imposible, bajo el mecanismo de competencia; siendo la cooperación la que facilita este tipo de vínculo.

En el caso del vínculo multiempresa local bajo el mecanismo de competencia, éste dará paso a la formación de una red local en la medida en que más empresas se agreguen al mismo. En otras palabras, las redes locales se formarán sin importar demasiado que se caractericen por estrategias de competencia o de cooperación. Esto se logra porque como ya se ha mencionado existen fuerzas centrípetas (Krugman, 1996) en la localidad que son independientes al mecanismo de cooperación. En otras palabras, en la medida en que más empresas forman parte de un vínculo multiempresa es mas difícil concebir un objetivo bajo el mecanismo de cooperación, dando paso al establecimiento de vínculos de competencia y de jerarquías entre las empresas.

El vínculo multiempresa local presenta la oportunidad para la formación y fortalecimiento de las redes locales. Además, a través de este vínculo el empresario experimenta la negociación alternando entre las estrategias de cooperación y de competencia, evaluando resultados y aprendiendo de los errores y conflictos ante actos oportunistas. Todo esto le prepara para el establecimiento de vínculos con empresarios extranjeros.

La búsqueda de socios extranjeros exige un mayor esfuerzo que, como señala Koschatzky (2002), a menudo sólo podrá ser llevado a cabo por las empresas que cuenten con los recursos de información y conocimiento apropiados. Como él mismo señala: cuanto mayor es la base de conocimiento de una empresa y cuanto 
REDES- Revista hispana para el análisis de redes sociales

Vol. 14,\#10, Junio 2008

http: // revista-redes.rediris.es

más desarrolladas sean sus competencias empresariales para integrar conocimientos externos a ella (Le Bars, et al. 1998: 316) tanto más desarrollada estará su capacidad para absorber nuevos conocimientos y, en consecuencia, para innovar. Creemos que los vínculos múltiples locales presentan un espacio para que el empresario se forme en este sentido, que aprenda a evaluar cuando cooperar y cuando competir y a enfrentar el oportunismo. Al mismo tiempo que al adiestrarse en el establecimiento de vínculos pretenda establecerlos con el extranjero.

Estudiar el vínculo para determinar si se estableció bajo el mecanismo de competencia o de cooperación exige investigación de orden cualitativo, que en ocasiones puede prolongarse bajo un periodo de varios años.

Para comprender esto último y analizar cómo se da el aprendizaje en los casos presentados es importante señalar las características típicas de las redes, las cuales son (Fritsch 2001, Powell 1990, Koschatzky 2000): la baja dependencia de los socios frente a la red, la falta de especificidad de las relaciones de intercambio, la ausencia de relaciones jerárquicas, la reciprocidad de las relaciones, la temporalidad de las relaciones, la alta flexibilidad ante la ausencia de contratos, la menor burocratización y necesidad de control, economías de escala y la confianza.

Además, dado que las empresas no disponen de una única opción para adquirir recursos, es grande la posibilidad de que éstas participen en varias redes de manera simultánea, siendo esto lo habitual para ellas (Koschatzky, 2002).

Por otra parte, las situaciones de fracaso en una red pueden ser una ventaja para el empresario cuando aprende de ellas. Sin embargo, el coste de esta forma de aprendizaje puede ser elevado, sobre todo en aquellos casos en los que existe una fuga incontrolada de conocimientos y de competencia tecnológica.

El vínculo multiempresa local bajo el mecanismo de cooperación fortalece la red. La red local requiere estar fortalecida para que al insertarse una o más empresas de la misma en la red de cooperación empresarial internacional puedan provocar y prolongar el desarrollo local. En caso contrario, existe la probabilidad de que la empresa inserta en el ambiente internacional se desprenda de la localidad, sin traer beneficio alguno o empobreciéndolo.

Para Sáez y Cabanelas (1997) las redes de cooperación son la expresión organizativa de los acuerdos entre empresas. Desde nuestra perspectiva, una red 
REDES- Revista hispana para el análisis de redes sociales

Vol. 14,\#10, Junio 2008

http: // revista-redes.rediris.es

de cooperación empresarial es la expresión organizativa de los vínculos de cooperación.

Por medio de los vínculos de cooperación se pueden crear nuevos entramados entre empresas de menor dimensión que exceden a lo local y que se sitúan en un momento determinado en el ámbito internacional. A este conjunto de entramados es a lo que denominamos red de cooperación empresarial internacional. Dicha red permite a las empresas de menor tamaño actuar más allá de su ámbito local, además de lograr sinergias que de otra manera resultarían imposibles actuando solas.

Las redes locales se mantienen aún cuando se caractericen por una mayor existencia de vínculos de competencia porque existen fuerzas centrípetas independientes a dichos vínculos que mantienen a las empresas concentradas geográficamente (Fujita, Masahisa, Krugman y Venables 2000). Contrario a esto último, los vínculos de competencia debilitan las redes de cooperación empresarial internacionales -en las que las empresas están geográficamente alejadas- y pueden provocar su desaparición porque en estas redes las fuerzas centrífugas son mayores a las centrípetas, siendo entonces los vínculos de cooperación fundamentales para mantener dichas redes (González 2007).

Las redes locales en las que los vínculos de competencia imperen tenderán a debilitarse en la medida en que las empresas que le conformen se internacionalicen (De Martino, et al 2006). Contrario a esto último, aquellas redes en las que la cooperación impere se fortalecerán y beneficiarán de la inserción internacional de alguna de sus empresas. En el primer caso, las empresas verán menos atractiva la vinculación local en la medida que consigan relaciones con empresas extranjeras. En el segundo caso, la empresa considerará fundamental la vinculación con la localidad porque dicha vinculación bajo el mecanismo de cooperación permite tomar conciencia de la comunidad y sus necesidades.

Las redes de cooperación empresarial internacionales, a diferencia de las redes locales de empresas, han vencido los límites en espacio. Esto lo decimos porque a través de los encuentros empresariales ${ }^{5}$ (Al-partenariat, Asia-Partenariat, Europartenariat, entre otros) las empresas entran en contacto con empresas de otros países sin necesidad de viajar a cada uno de éstos. También vencen el límite

\footnotetext{
${ }^{5}$ Entrevistas cara a cara entre empresarios que pertenecen al mismo sector de negocios; o bien, a sectores complementarios.
} 
REDES- Revista hispana para el análisis de redes sociales

Vol.14,\#10, Junio 2008

http: // revista-redes.rediris.es

del tiempo al permitir que bajo los vínculos de cooperación una empresa se retroalimente con la experiencia de otra (González 2003).

Las redes de cooperación empresarial se forman con base en vínculos caracterizados por el mecanismo de cooperación: crear valor y compartirlo. Los vínculos en la red internacional se establecen, no necesariamente por la cercanía física, ni por la espontaneidad del interactuar cotidiano, más bien, por la convicción que los empresarios tienen por cooperar. Estos empresarios probablemente pertenecen a distintos países o regiones, hablan otro idioma y, en algunos casos, han asistido a encuentros empresariales. Pero cabría preguntarnos ¿de qué manera el empresario se convence de que la cooperación permite obtener, en un momento determinado, mayores ventajas que la competencia? El aprendizaje local, en ocasiones, permite al empresario evaluar ante la disyuntiva de cooperar o competir e inclinarse cada vez más hacia la cooperación cuando se trata de vincularse con empresas extranjeras complementarias.

En relación con esto último, se desarrolló un trabajo de investigación, cuyo análisis se centra en los encuentros empresariales. Estos últimos han sido renombrados con el término "clusters temporales". En el estudio mencionado sólo se analiza el momento en que las empresas se encuentran reunidas bajo el encuentro empresarial. No se hace un seguimiento de las mismas, posterior al encuentro, para con ello observar qué resultados se obtienen a mediano plazo (Maskell et al. 2006). Es pertinente estudiar cómo las empresas, después de dichos encuentros, crean, mantienen o terminan los vínculos empresariales. Este estudio nos conduce a diferenciar las redes locales de las internacionales y a definir los vínculos multiempresa como articuladores de ambas redes.

Analizar el vínculo multiempresa, la cooperación como mecanismo y la articulación a la red internacional, con base en la evidencia empírica permite señalar las ventajas y desventajas que esto implica para las empresas de menor dimensión. A continuación hacemos referencia a los resultados generales del proyecto y a la manera en que el análisis de cinco casos permite fundamentar lo dicho en los párrafos anteriores. El primer caso para analizar corresponde a un vínculo multiempresa que, al insertarse en una red internacional, ha logrado beneficiar a cuarenta y cuatro comunidades indígenas en México; este primer caso representa un vínculo múltiple que ha permitido articular una red local con una internacional, además, la red local se encuentra no sólo geográficamente dispersa, sino conformada por grupos con diferente idioma. El segundo caso se refiere a otra empresa que por medio del vínculo múltiple ha logrado acumular el aprendizaje 
REDES- Revista hispana para el análisis de redes sociales

Vol.14,\#10, Junio 2008

http: // revista-redes.rediris.es

suficiente para actuar en el mercado asiático y europeo, beneficiando con ello a más de 270 pequeños productores. El tercer caso presentado es sobre un vínculo en el que impera el mecanismo de competencia, por tanto, la empresa ha logrado establecer relaciones asimétricas con empresas trasnacionales que actúan en el país, pero no así con el extranjero; además, el hecho de que el mecanismo de competencia impere, no ha permitido beneficiar a la localidad de la misma forma que se beneficiaría bajo el mecanismo de cooperación. El cuarto caso es sobre una empresa que mediante la cooperación local enfrentó y sobrevivió a la crisis en Argentina, transitando de las estrategias de competencia hacia las de cooperación, estableciendo con ello un vínculo en el que la centralidad tiende a disminuir, este caso nos muestra que una empresa que actúa en forma reactiva ante los cambios externos, en ocasiones, establece un vínculo multiempresa. El quinto caso seleccionado es una empresa europea del siglo XVIII que logró participar en el ámbito internacional a través de los vínculos de cooperación, creemos que es importante traer este caso a nuestro análisis porque estamos hablando de otro contexto histórico y económico.

\section{Resultados generales de investigación y análisis de casos}

En el estudio del cual se deriva el presente artículo, se ha hecho el seguimiento a un grupo de empresas del sector agroindustrial mexicano. Este seguimiento es sobre los vínculos establecidos por un periodo que inició en el 2003 hasta el día de hoy. Para recuperar la evidencia empírica se ha contado con los siguientes instrumentos: observación directa en un encuentro empresarial al que las empresas asistieron en el 2003 (202 empresas agroindustriales), una encuesta aplicada en el 2004 (129 empresas), un cuestionario aplicado en el 2005 (86 empresas), visitas de campo a las empresas durante el periodo 2006-2008 y; una encuesta aplicada por teléfono en el 2008 (42 empresas ${ }^{6}$ ).

Entre los resultados preliminares sobre las cuarenta y dos empresas se tienen los siguientes: el ochenta por ciento de los empresarios participan en encuentros empresariales de manera constante; el setenta y uno por ciento mantienen vínculos fuera de su localidad, ya sea con el extranjero o con empresas ubicadas en otras partes del país; el cincuenta y dos por ciento ha enfrentado el fracaso de un vínculo empresarial; el noventa y tres por ciento expresó interés por continuar estableciendo nuevos vínculos, dentro de este porcentaje se encuentran los que 
REDES- Revista hispana para el análisis de redes sociales

Vol.14,\#10, Junio 2008

http: // revista-redes.rediris.es

han pasado por una experiencia de fracaso. Ninguno de los empresarios que han afrontado un fracaso en el establecimiento de vínculos expresó no estar interesado en establecer nuevos vínculos. Esto último muestra que dichas experiencias, si bien son negativas y pueden representar un alto costo para la empresa, también son una forma de aprender y no desmotivan al empresario ante la idea de establecer nuevos vínculos. Por otra parte, de los 42 contactos, tres de ellos han cerrado su empresa y creado una nueva, lo que muestra que los empresarios que logran establecer vínculos de manera constante son receptivos a los cambios y están dispuestos a aprender de los errores, aún en el extremo de cerrar la empresa.

De este grupo de empresas $y$, tras el seguimiento por varios años, se han seleccionado aquellas que cumplen con el perfil de vínculo múltiple (vínculo multiempresa). Para el estudio de los vínculos multiempresa nos hemos valido de las visitas de campo, entrevistas y llamadas por teléfono que facilitan el seguimiento de los mismos. En el presente trabajo hemos traído a discusión cinco casos, tres de ellos derivados del estudio, dos más se han recuperado a través de la literatura respecto al tema. El primer caso es "Maíz y café", un vínculo múltiple que fortalece la red local y la articula a una internacional, beneficiando a la población; el segundo caso es "La cooperativa de Aguacate", vínculo múltiple que fortalece a la red local, favoreciendo a la población por su presencia en los mercados asiático y europeo; el tercer caso "Nuez de la discordia", es un vínculo múltiple en el que impera el mecanismo de competencia, frenando con ello el desarrollo local, a pesar de que el producto cuenta con una demanda nacional y con posibilidades de exportar; el cuarto caso es "Zanon", vínculo de carácter local que permite analizar el mecanismo de cooperación ante una crisis económica; y finalmente "Paños catalanes", vínculo que en otro contexto histórico-económico permitió fortalecer la red local y articularla a una internacional.

\section{a) Caso I. "Maíz y Café", propiciando el desarrollo local}

La empresa, a la que llamaremos "Maíz y café"”, ubicada en el estado de Oaxaca, podría considerarse como la expresión de un vínculo multiempresa que sirve de pilar para una red local. "Maíz y café" está formado por empresarios de diferentes grupos indígenas.

\footnotetext{
${ }^{6}$ El número de empresas es hasta el día 17 de junio de 2008, recordando que puede aumentar por derivarse de un proyecto en marcha.

${ }^{7}$ Los nombres de las empresas correspondientes a los tres primeros casos han sido modificados a fin de conservarles en el anonimato.
} 
REDES- Revista hispana para el análisis de redes sociales

Vol.14,\#10, Junio 2008

http: // revista-redes.rediris.es

La identidad de las personas y de los grupos o comunidades en México es dinámica y continuamente se redefine, incluso puede modificarse. Por ello es importante comprender que las poblaciones indígenas son un conjunto de pueblos y personas que mantienen una enorme diversidad en historias, lenguas y culturas, poseen maneras propias y diversas de ver y entender el mundo, así como formas específicas de organización y cohesión social. La cultura de los pueblos indígenas no es homogénea ni estática, concebirlos de esta manera, nada tiene que ver con su realidad social e histórica (Suárez, 2006: 85). Bajo esta óptica analizaremos este vínculo multiempresa.

"Maíz y café" está formado por pequeños productores y tiene veintiún años de existencia. Dentro de la región hay comunidades que se pueden considerar inaccesibles por no contar con vías terrestres, en las que los productores caminan hasta ocho horas por rutas difíciles, cargando 50 kilos en la espalda para transportar su producto a la ciudad más cercana y venderlo.

Estos productores, al unirse por medio de un vínculo múltiple han logrado, a través de los años, conformar una red local que les ofrece mayores oportunidades tanto para ellos como para los miembros de su familia. Esta red local al fortalecerse, vía la experiencia y el aprendizaje alcanzados por los productores dentro de un vínculo múltiple, se ha insertado a su vez en una internacional. La red de cooperación empresarial internacional a la que se insertó es provocada por Comercio Justo. Mediante esta red internacional "Maíz y café" participa en los mercados extranjeros, permitiendo así que productores indígenas comercialicen café, maíz, aguardiente, piloncillo, entre otros más. Todos estos productos son enviados y promovidos en el extranjero como orgánicos.

Las empresas locales ${ }^{8}$ durante décadas han cooperado, compartiendo los clientes y transmitiendo unas a otras las técnicas de cultivo y cualquier otro tipo de información que permita mejorar sus productos e innovar en armonía con el desarrollo sustentable. La empresa con mayor experiencia tiende a enseñar y apoyar a las nuevas empresas. En el caso de "Maíz y café", se ha beneficiado del apoyo de otra empresa hermana que cuenta con diez años más de antigüedad, que ha compartido clientes, que ha compartido capacitación y en ocasiones recursos.

\footnotetext{
${ }^{8}$ Nos referimos a las empresas que se encuentran en las mismas comunidades indígenas con productos similares a los "Maíz y café".
} 
REDES- Revista hispana para el análisis de redes sociales

Vol.14,\#10, Junio 2008

http: // revista-redes.rediris.es

La red local sostenida por el vínculo múltiple cuenta con un sistema de comunicación eficiente - las comunidades trabajan y aprenden a pesar de la lejanía entre ellas y de los distintos idiomas- que permite transmitir la capacitación de los representantes a cada miembro de las diferentes comunidades, con lo que se reducen costos y se obtienen mayores beneficios derivados de la misma. Los representantes de "Maíz y café" cuentan con una visión internacional, con un sentido de protección al ambiente, con apoyo hacia la comunidad y con respeto a la madre tierra. Ellos consideran que "el respeto a la madre tierra" es importante para aumentar la calidad de vida.

Las reuniones generales en "Maíz y café" se llevan acabo en español, a pesar de que la asamblea se conforma por personas que hablan mixteco, mixe, chinanteco, cuicateco, zapoteco y chatino. Para salvar los problemas de comunicación en las comunidades cuando hay intercambio entre las mismas se cuenta con traductores.

Los representantes de "Maíz y café" han participado en encuentros empresariales en Alemania y Estados Unidos, siempre con el apoyo de los organismos internacionales, sin embargo, su principal respaldo es la misma red. La red de Comercio Justo abre a los empresarios insertos en la misma el mercado internacional en un ambiente concertado. Esto último permite a las empresas participar en el extranjero sin las barreras que por lo común se enfrentan los pequeños empresarios (normas internacionales, proteccionismo, competidores en el país destino, entre otras). En el momento en que Comercio Justo los certifica se abre un canal directo al mercado extranjero para el vínculo múltiple "Maíz y café" y, con ello, para la red local que dicho vínculo sostiene en las cuarenta y cuatro comunidades.

La competencia que "Maíz y café" enfrenta es local y provocada por los intermediarios. Estos últimos llegan a la puerta del pequeño productor y le ofrecen la compra de sus productos sin considerar la calidad de los mismos; a su vez, el intermediario vende este producto a las trasnacionales. Esta práctica intermediaria inhibe el estímulo a los pequeños productos para que aumenten la calidad; provoca la caída de los precios en el nivel local y con ello de las ganancias; además de boicotear el proyecto para el desarrollo que lleva acabo Comercio Justo a través de las empresas insertas en su red. Es importante señalar que de no existir vínculos multiempresa, en la localidad, todos quedarían expuestos al mecanismo de competencia usado por las trasnacionales. 
REDES- Revista hispana para el análisis de redes sociales

Vol.14,\#10, Junio 2008

http: //revista-redes.rediris.es

Si analizamos a "Maíz y café" con base en lo expuesto en la primera parte del artículo entonces le podemos calificar como un vínculo multiempresa. Vínculo que se ha convertido en el pilar y catalizador de una red local (1185 socios), en un espacio de aprendizaje para los implicados y como un agente articulador entre una red local y una internacional. La red que conforman los productores de las cuarenta y cuatro comunidades de siete regiones de Oaxaca puede considerarse como local desde el punto de vista geográfico; sin embargo, al considerar, tanto la baja infraestructura en comunicaciones como la estructura multicultural y plurilingüe de los vinculados, se puede definir como red internacional. Esto último en el sentido de que dicha red para su sostenimiento requiere de la existencia de vínculos de cooperación entre los productores participantes. Esta red local se encuentra articulada a una red internacional, siendo el vínculo multiempresa representado por “Maíz y café" el que funge como articulador.

\section{b) Caso II. “La cooperativa de aguacate" ante el oportunismo}

La cooperativa ubicada en Tacámbaro, México está formada por 270 pequeños productores de aguacate, quienes lo comercializan en forma conjunta. Esto último disminuye la existencia de intermediarios, permitiendo que los costes de comercialización no sean elevados, al mismo tiempo que permite que las ganancias sean mayores para cada productor. El aguacate lo exportan a Canadá, Francia, Japón y Costa Rica.

Este vínculo multiempresa es un claro ejemplo de que los sistemas productivos locales aumentan la posibilidad de prevalecer si se articulan a redes de cooperación empresarial internacionales. La cooperativa con más de cincuenta años ha aumentado su producción, y garantizado la demanda de su producto en el nivel internacional, al vincularse con empresas extranjeras. El impacto económico en la localidad ha sido positivo, contribuyendo a mejorar el nivel de vida de los agricultores, abriendo la oportunidad para que los hijos de estos tengan una mejor educación y colaborando con el municipio para la creación de una infraestructura más favorable, lo que incluye el mantenimiento en buen estado de los caminos carreteros que conducen a esta localidad.

En el caso de la cooperativa, al romperse un vínculo de cooperación con una empresa francesa, la reacción de los representantes fue buscar lo más pronto posible un nuevo vínculo con otra empresa del mismo país. El ingeniero durante la entrevista expresó la necesidad de que la cooperativa se mantuviera en el mercado francés, reconociendo la importancia de que la empresa se internacionalice. 
REDES- Revista hispana para el análisis de redes sociales

Vol.14,\#10, Junio 2008

http: // revista-redes.rediris.es

Desde el punto de vista del ingeniero mexicano, el empresario francés no tuvo la suficiente sinceridad para reconocer que el mercado se había cerrado, y con ello, ambas partes compartir las pérdidas. El empresario francés simplemente no pagó el último pedido que se le había enviado, esperó a que existiese un reclamo por parte de los tacambarenses, y finalmente, declaró que el cliente final había regresado el producto por defectos en el mismo.

Por ser el aguacate un producto perecedero no hay forma de demostrar que el producto estaba dañado, ni de demostrar que el producto estaba en buenas condiciones. Tampoco existe un medio seguro para determinar quién fue el verdadero responsable: el mexicano, el francés o el vendedor final. Ante situaciones como esta, en circunstancias llenas de incertidumbre, es muy difícil llegar a un acuerdo formal que establezca una solución justa para todas las partes implicadas. Mucho más difícil resulta establecer mecanismos que permitan cubrir de este tipo de riesgo a los exportadores de productos frescos.

Esta experiencia mostró lo frágil que es para los productores la venta hacia el extranjero; sin embargo, esta circunstancia no hizo que se desistieran de establecer vínculos con el extranjero.

Es probable que la experiencia dentro del vínculo multiempresa que localmente han establecido haya influido decisivamente en el comportamiento de la cooperativa ante el conflicto con la empresa extranjera. Es decir, ante el conflicto "cooperativa de aguacate" no se centró en buscar quién era el culpable, más bien, buscó una posible solución que mantuviera a la empresa en el mercado internacional.

También es probable que se tuviese claro que la otra empresa no era el mercado meta, era un medio para llegar a su mercado. Por tanto, al romperse el vínculo no se consideró que el mercado francés se había cerrado y se decidió como alternativa encontrar una nueva empresa que les permitiese mantener a la cooperativa exportando al mercado francés.

Por otra parte, durante un encuentro empresarial en Canadá se estableció un vínculo de cooperación con un transportista mexicano-canadiense que permitió a la cooperativa entrar a este mercado. El ingeniero reconoció que fue a Canadá sin vender nada pero que estableció una relación con el transportista que le permitió entrar al mercado. Ante estas circunstancias y por iniciativa propia estableció vínculos fuera del encuentro empresarial con una empresa canadiense a la que le envía medio contenedor de aguacate cada semana por medio del transportista.

c) Caso III. "Nuez de la discordia", el vínculo de competencia como freno del desarrollo local 
REDES- Revista hispana para el análisis de redes sociales

Vol.14,\#10, Junio 2008

http: // revista-redes.rediris.es

Este vínculo multiempresa nació con el apoyo de Fonaes (Fondo Nacional de Apoyo a las Empresas Solidarias, México), en sus treinta años de vida ha transitado del mecanismo de cooperación al de competencia. Siendo su primera etapa la de mayor crecimiento y beneficio local. Los miembros del vínculo se unieron bajo el mecanismo de cooperación, crear valor y compartirlo. Así, mientras uno puso su terreno, otro instaló un cuarto en frío, otro más inició los trámites ante Fonaes, y no faltó quien capacitara y enseñara el cultivo y proceso de la nuez asiática a los productores locales.

El vínculo surge con pequeños productores de nuez, un grupo de mujeres indígenas que se encargan de procesarla y una familia con ascendencia francesa que se estableció en la localidad. Todos ellos, tras aprender a producir dicho fruto, encontraron una mejor alternativa de vida. Alternativa que de permanecer bajo el mecanismo de cooperación habría traído mayores beneficios a la localidad, como es el caso de "Maíz y café" y de "La cooperativa de aguacate".

En este caso podemos observar la transición del vínculo de cooperación a uno de competencia. Dicha transición no se dio de manera rápida, fueron los años los que entretejieron los hechos y fomentaron la desconfianza. Los hilos conductores fueron: la búsqueda de niveles jerárquicos, el beneficio a corto plazo, la falta de comunicación entre las partes y la muerte del socio con mayor edad. La pérdida de alguien que tenía cierta representatividad y admiración dentro del vínculo condujo a mayores distanciamientos y a conflictos internos, incluso de carácter legal.

Estos conflictos han abandonado el seno del vínculo, han trascendido en lo local, conduciendo a que los pequeños pobladores tomen partido en los mismos, creando un ambiente hostil entre la población.

El resultado de todo esto es que el vínculo se mantiene operando en el nivel nacional por una sobre demanda del producto. El vínculo multiempresa ha establecido relaciones asimétricas con empresas trasnacionales que operan en el país, sin darse tiempo para el establecimiento de vínculos más equitativos con empresas de similar tamaño de otras regiones o países. Tampoco se ha dado tiempo para aumentar el número de socios y con ello extender los beneficios en la región.

La sobre demanda de la que aparentemente disfrutan es porque pocos son los productores nacionales que pueden garantizar la calidad de la nuez durante todo el año. Sin embargo; los conflictos internos frenan el aprendizaje de los miembros, distraen de las tareas de vinculación hacia el exterior y desvían los esfuerzos que pudieran darse con miras a aumentar el número de socios. 
REDES- Revista hispana para el análisis de redes sociales

Vol.14,\#10, Junio 2008

http: // revista-redes.rediris.es

Este vínculo multiempresa es un claro ejemplo de los resultados que se obtienen bajo el mecanismo de competencia, si bien la empresa se encuentra operando en el nivel nacional, no se han obtenido los resultados locales que se esperarían si estuviese bajo el mecanismo de cooperación.

\section{d) Caso IV. "Zanon" y el mecanismo de cooperación ante la crisis}

Kilkeny y Nalbarte (2003) han analizado las redes que se crean dentro de una comunidad, es decir, la forma como las organizaciones en una comunidad se relacionan, y como estas relaciones se mantienen y fortalecen por medio de lo que pudiera llamarse capital social. Capital que al analizarse desde la perspectiva de la red, estos autores lo denominan Capital de la red comunitaria (community network capital). Es interesante ver cómo una comunidad con elevada confianza en sus organizaciones tiende a crecer más y estará mejor preparada para auto-organizarse ante una eventualidad.

En el proyecto, estos estudiosos centran su atención en el sector clave (Keystone sector). Refiriéndose con el término sector clave a la entidad sin la cual la estructura de la red sería profundamente alterada. En otras palabras, en aquella entidad que permite mantener la estructura de la red porque goza de mayor centralidad y prestigio (Kilkeny, 2005; Kilkeny y Nalbarte, 2003). El caso de Zanón nos muestra cómo una empresa puede convertirse en el sector clave.

Es importante subrayar que Argentina ha tenido que enfrentar un período caracterizado por fuertes turbulencias macroeconómicas. El término del tipo de cambio fijo se produjo en el marco de una profunda crisis económica y social que fue acompañada por el default de la deuda pública y una parte sustantiva de la deuda privada, el congelamiento de los depósitos bancarios, fuertes restricciones en el circulante y la desaparición del crédito para los sectores productivos (Dini et al. 2005). Bajo estas condiciones se creó éste vínculo multiempresa.

La fábrica de cerámicas Zanon está localizada en la provincia de Neuquén, en una región de la Patagonia y es una de las principales fábricas de azulejos para suelo y pared en Argentina. Fue inaugurada en 1979 con cuatro líneas de producción, gracias a una subvención de terreno gratuito, créditos estatales, exenciones fiscales y tarifas de gas y eléctricas a bajo precio. Cuando los obreros la tomaron en 2002, su deuda total con diversos acreedores públicos y privados ascendía a 75 millones de dólares (Petras y Veltmeyer, 2004: 215). Estos trabajadores distribuyeron volantes bajo el lema “Una fábrica dirigida por los trabajadores al servicio de la comunidad". 
REDES- Revista hispana para el análisis de redes sociales

Vol.14,\#10, Junio 2008

http: // revista-redes.rediris.es

La planta emplea a trescientos diez trabajadores, de quienes dependen económicamente mil quinientas personas. La política de los trabajadores consiste en "comprar material local" en la medida de lo posible: materias primas, piezas de maquinaria, alimentos, ropa de trabajo, entre otros. El poder adquisitivo de los trabajadores ha estimulado pequeños comercios locales (Petras y Veltmeyer, 2004: 216).

El éxito de los trabajadores de Zanon en el mantenimiento del control y en la continuación de la producción se debe a varios factores: establecieron vínculos con estudiantes, grupos religiosos, organizaciones de trabajadores desocupados y diversos sindicatos (maestros, funcionarios, profesores de universidad); desarrollaron un alto grado de solidaridad y de conciencia de clase ante la toma del establecimiento al grado que "ninguna personalidad domina las reuniones o asambleas"; además, han aprendido lo que no sabían en el manejo de la fábrica (Petras y Veltmeyer, 2004: 217). Estos vínculos se establecieron por el nivel elevado de confianza que la comunidad a mostrado hacia Zanón. Disfrutando esta última del prestigio y centralidad correspondientes a un sector clave (Kilkeny y Nalbert, 2003).

La situación en la que se encuentra la fábrica no es nada fácil. Sin embargo, es importante hacer notar el establecimiento de un vínculo múltiple que en el nivel local ha permitido crear sinergia. Este hecho es lo que logró que la fábrica continúe operando en medio de la inestabilidad económica y política argentina.

Podemos decir que el vínculo múltiple creado por Zanon posiblemente es de cooperación (crear valor y compartirlo). Esto se deduce por las características que presenta: la empresa trabaja bajo el lema “Una fábrica dirigida por trabajadores al servicio de la comunidad"; priorizan la compra de material local; y, ninguna "personalidad" domina en las reuniones. Además, el aprendizaje y el trabajo voluntario para crear valor se pueden considerar como características principales del vínculo establecido por Zanon con las organizaciones locales.

La cooperación abre la puerta a la adaptación y el cambio antes que a la permanencia. Todo ello ha conseguido que la historia de esta fábrica argentina, en medio de la inestabilidad, sea marcadamente diferente a sus contemporáneas. Zanon se ha convertido al paso del tiempo en un vínculo múltiple que, al actuar en la localidad, ha servido de apoyo para la creación y mantenimiento de una red a la que se han sumado otras empresas como proveedoras y diferentes organizaciones. Sin embargo, existe la posibilidad de que este vínculo múltiple busque contrapartes 
REDES- Revista hispana para el análisis de redes sociales

Vol. 14,\#10, Junio 2008

http: //revista-redes.rediris.es

en el extranjero para con ello articular la red local a una internacional. Esto último prolongará los beneficios que localmente se han obtenido por las vinculaciones de Zanon.

\section{e) Caso V. "Los paños catalanes" participando en mercados internacionales}

En la España del siglo XVIII era todavía muy notoria la actividad de las "diásporas mercantiles" o redes comerciales integradas. Estas redes comerciales las formaban mercaderes extranjeros, quienes inundaban España con sus productos. Como bien señala Torras (1995: 113) esto no era una manifestación de apertura económica, sino más bien de lo contrario. La actividad económica tiende a organizarse de ese modo en una economía atrasada, con escasa integración de mercados.

En este marco económico Torras estudia un sector tradicional de la economía catalana, la manufactura de paños, en el cual la protección arancelaria fue poco efectiva durante la mayor parte del siglo y en el que la intervención del Estado tuvo importancia secundaria. (Torras, 1995: 114).

I gualada era uno de los mayores centros de fabricación de paños de Cataluña hacia 1760. Torras expone con cierto detalle la aparición de una empresa pañera familiar en uno de los centros textiles y propone algunas explicaciones de su éxito, haciendo hincapié en la vertiente mercantil de la empresa. Se trata de una empresa artesana, en un mercado interior, que creció a base de aumentar sus ventas fuera de Cataluña, en mercados donde operaban diásporas mercantiles bien arraigadas. En este caso se destaca la capacidad del empresario para establecer su propia diáspora mercantil en otras regiones de España. Esto último es considerado como un factor a tener en cuenta al explicar el éxito de la industria catalana para vender en mercados entonces poco protegidos y en los que los productos y comerciantes extranjeros ocupaban posiciones sólidas.

Entre las familias Torelló y Borrull existían matrimonios cruzados que garantizaban una cooperación estable entre las dos familias artesanas, con la siguiente puesta común de información, relaciones e influencias y, asimismo, de recursos técnicos. También se fortalecía la confianza mutua requerida para compartir gastos en inversiones fijas y asumir los riesgos de la comercialización en mercados distantes. Borrull y Torelló distribuían fibra para hilar a 340 hilanderas, y diecinueve telares anchos trabajaban continuamente para ellos; en total, daban ocupación directamente a 500 personas. En 1765 el número de telares ocupados ascendió a veintisiete (Torras, 1995: 119-122). 
REDES- Revista hispana para el análisis de redes sociales

Vol.14,\#10, Junio 2008

http: // revista-redes.rediris.es

Los estambres para urdir se compraban en localidades que se habían especializado en esta producción, como la Pola de Lillet, localidad en la que la hilatura de lana peinada se había convertido en importante fuente de ingresos para muchas familias en una comarca montañosa de limitados recursos agrícolas. Pero casi toda la lana (de carda) la hilaban en sus casas mujeres y niñas que vivían alrededor de I gualada, en un radio de quince a veinte kilómetros. Se puede conjeturar que Torelló subcontrataba a pelaires de menos vuelos las tareas de distribuir la fibra y recoger las hilazas (Torras, 1995: 128).

El éxito de los Torelló y Borrull, de acuerdo con Torras (1995: 123,127) se debe a la capacidad para establecer su propia red de distribución y, eliminando intermediarios, reducir los costos de comercialización y hacer más competitivos sus géneros; además, la conciencia de ser diferente fortalecía la cohesión del grupo, que daba lugar a lazos de confianza mutua y proporcionaba canales exclusivos de información, poderosas herramientas para lograr el éxito comercial en un entorno poco favorable.

En el caso de Torelló y Borrull podemos ver el caso de un vínculo local que a través del tiempo fue fortaleciendo la confianza para, con ello, dar paso al establecimiento de vínculos de cooperación con terceros. Este vínculo permitió beneficiar a las entidades económicas locales al crear y mantener una red de proveedores y colaboradores que con el tiempo se fortaleció y extendió. El beneficio local se prolongó con el establecimiento de nuevos vínculos en el exterior. De tal forma que el vínculo Torelló y Borrull se convirtió en articulador de la red local a una internacional (considerando que participaban en mercados distantes). En este caso, se puede observar que los vínculos múltiples propician el desarrollo local y lo prolongan cuando actúan como articuladores.

\section{Conclusiones}

Los casos presentados muestran afinidades y diferencias que vale la pena analizar con base en el primer apartado, en el que se expusieron los aspectos teóricos. Entre las afinidades con las que se cuenta son: la existencia de un vínculo múltiple, en cuatro de los casos son multiempresas; no así en el caso de Zanón, en el que sería un vínculo multi-organizaciones. Esto último porque en el vínculo se encuentran involucrados diferentes organizaciones, no sólo empresas.

En los cinco casos se muestra la importancia de la confianza y, con ello, del mecanismo de cooperación para lograr mayores beneficios para las partes 
REDES- Revista hispana para el análisis de redes sociales

Vol.14,\#10, Junio 2008

http: // revista-redes.rediris.es

involucradas. Cuatro de los casos han transitado del mecanismo de competencia al de cooperación, sólo en el caso de "Nuez de la discordia" observamos lo contrario. Con excepción de "Nuez de la discordia", en los cuatro casos la centralidad hacia dentro del vínculo ha disminuido, eliminándose las jerarquías dentro del mismo; a su vez, la centralidad de estos vínculos en relación con las empresas que no forman parte de él ha aumentado.

En el caso de "Nuez de la discordia", la centralidad entre los pequeños productores que forman el vínculo se ha marcado, trayendo como resultado relaciones asimétricas con otras empresas y colocándose en situaciones poco ventajosas para este vínculo multiempresa.

En tres de los casos en los que el vínculo se estableció bajo el mecanismo de cooperación, "Maíz y café", "La cooperativa de aguacate" y "Los paños catalanes", estos vínculos han funcionado como articuladores de la red local a una internacional, prolongando los beneficios del desarrollo local.

Las ventajas y desventajas que las redes de cooperación empresarial presentan a las empresas pequeñas y medianas, conforme a los casos analizados son:

a) "Maíz y café" nos permite observar la manera en que un vínculo múltiple propicia el desarrollo en comunidades cuya infraestructura les hace casi imposible vincularse localmente y, con mayor razón, participar en mercados externos. Este vínculo múltiple ha logrado internamente vencer las diferencias culturales y lingüísticas; a su vez, ha prolongado el desarrollo local por más de veinte años al insertarse en una red de cooperación empresarial internacional (Comercio Justo). Este mismo vínculo permite a los productores locales hacer frente a las estrategias de competencia que las trasnacionales provocan en la localidad.

Además, los productores en pequeño al vincularse con "Maíz y café" pueden hacer frente de manera colectiva a acuerdos con empresas extranjeras de mayor tamaño que de otra manera les colocarían ante una relación asimétrica. Relación asimétrica que por su propia naturaleza estaría caracterizada por estrategias de competencia y que al hacer sinergia entre los productores logran mejor negociación, inclinando la relación hacia estrategias de cooperación.

Por otra parte, el que “Maíz y café participe en una sola red internacional se convierte en una desventaja. La dependencia de un vínculo múltiple en una 
REDES- Revista hispana para el análisis de redes sociales

Vol.14,\#10, Junio 2008

http: // revista-redes.rediris.es

red es poco probable que se de. Lo común es que las empresas participen simultáneamente en más de una red. En este caso, se está dando dicha dependencia y esta representa el "Talón de Aquiles" de "Maíz y café" ¿Qué pasaría si Comercio Justo ya no operara? ¿Cuál sería el impacto si “Maíz y café" dejara de vincularse por medio de esta red?

b) En "la cooperativa de aguacate" podemos resaltar como ventajas de la red el que se prolongue el desarrollo local, mismo que pudiera haberse estancado de no haberse insertado en una red internacional. La cooperativa ha incursionado en regiones que por otra vía difícilmente habría tenido acceso. El que el vínculo múltiple se encuentre operando bajo el mecanismo de cooperación ha permitido que "la cooperativa de aguacate" no sólo enfrente el oportunismo derivado del producto que comercializa, sino que, en la medida que se internacionaliza fortalezca los vínculos locales. La desventaja de la red internacional en este caso se ve reflejada en los actos de oportunismo que pudieran darse al enviar productos frescos y perecederos al extranjero y que de hecho ya se han dado.

c) En relación con "Nuez de la discordia", la empresa no ha logrado insertarse en una red internacional, a pesar de asistir con cierta frecuencia a encuentros empresariales, ferias especializadas y a eventos gubernamentales creados para provocar el establecimiento de vínculos. El que en un vínculo exista el mecanismo de competencia frena la participación de este en otras redes, diezmando con ello los beneficios que loclamente pudieran obtenerse.

d) En relación con el caso de la empresa argentina, Zanon: pudimos corroborar que el establecer una red locla bajo el mecanismo de cooperación permitió que la empresa se mantuviera en operación, a pesar de las circunstancias. El crear un vínculo múltiple caracterizado por la solidaridad, la disposición al aprendizaje y al cambio y, con sentido de "comunidad", abrió paso al establecimiento de un vínculo de cooperación. Vínculo que primero permitió el inicio de la producción de la fábrica. Este mismo vínculo, al fortalecerse y establecer nuevas relaciones en la localidad permitió la formación de una red local, además, prolongó hasta el día de hoy la operación de la fábrica. Todo esto a pesar de las condiciones socioeconómicas adversas que Zanon enfrenta. La principal desventaja es que el vínculo múltiple no se ha insertado a una red internacional. En la medida en que éste se encuentre localizado, los beneficios irán menguando, 
REDES- Revista hispana para el análisis de redes sociales

Vol.14,\#10, Junio 2008

http: // revista-redes.rediris.es

aumentando los problemas financieros que enfrenta actualmente. Es un vínculo que no está articulando la red local a una internacional.

e) Con respecto al vínculo Torelló y Borrull, hemos visto un caso bajo un contexto histórico diferente en el que los empresarios, fortalecieron la red local y establecieron vínculos en regiones distantes. Siendo entonces el vínculo Torelló y Borrull un articulador de la red local a una internacional; al mismo tiempo que generó y aseguró 500 empleos de manera directa, con lo que posiblemente permitió mantener cierto nivel de vida en la localidad. Este vínculo caracterizado por las estrategias de cooperación representa a empresarios que han madurado en el uso de la red de cooperación empresarial internacional y, por tanto, permiten fortalecerla en la medida que estos mismos promueven la inserción de nuevas empresas.

Entre las desventajas que la red de cooperación empresarial representa a los empresarios son las siguientes:

La posibilidad de establecer una relación asimétrica con otra empresa que conduzca a la dependencia y, con ello, a menores beneficios de los que se obtendrían en una relación más equitativa.

El establecer un vínculo bajo el mecanismo de competencia y que, en un momento determinado por las circunstancias, el empresario se vea obligado a continuar con la relación.

La empresa puede exponerse a la fuga incontrolada de conocimientos y de competencia tecnológica.

Las redes internacionales por sí mismas tienden a ser frágiles, sobre todo si consideramos el factor tiempo, la distancia y el que en el presente sistema el mecanismo de competencia es el que permea los negocios en el nivel internacional.

Consideramos importante ampliar el estudio hacia otros sectores de negocio, tales como: medioambiente, automotriz y telecomunicaciones. Esta ampliación es para comparar los resultados entre sectores. Al mismo tiempo, resulta conveniente profundizar en el estudio de los vínculos para con ello explicar la reproducción de los vínculos, si la desaparición de un vínculo conduce a la creación de una red local, o bien, a la desaparición de aquella a la que se encuentra articulado. 
REDES- Revista hispana para el análisis de redes sociales

Vol. 14,\#10, Junio 2008

http: // revista-redes.rediris.es

Finalmente, el localizar los vínculos múltiples que están articulando las redes locales a las internacionales para estudiarlos permite reunir evidencia empírica que contribuya a que más empresas pequeñas y medianas actúen como articuladoras al fungir como vínculos múltiples bajo el mecanismo de cooperación.

\section{Bibliografía}

Barney, J. (1991). "Firm Resources and Sustained Competitive Advantage". Journal of Management, n1 17, pp. 99-110.

Casanueva, C. et al. (2003). “Relaciones estratégicas entre Pymes: contraste de hipótesis empresariales mediante ARS". Revista Hispana para el análisis de redes sociales, Barcelona, vol. 4, n. 4, junio.

Casanueva, C. et al. (2006). "Capital social, confianza e innovación. El caso de un sistema productivo local tradicional". Revista Madrid, №. 36.

Contractor, N. et al. (2006). “Testing Multitheoretical, Multilevel Hypotheses about Organizational Networks: An Analytic Framework and Empirical Example". Academy of Management Review, vol. 91, n. 3, pp. 691-703.

De Martino, R. et al. (2006). "Balancing localization and globalization: exploring the impact of firm internationalization on a regional cluster". Entrepreneurship and Regional Development, vol. 18, n. 1 pp. 1-14.

Dini, M. et. al (2005). El aporte de FUNDES a las pymes en América Latina: una evaluación de impacto, Santiago de Chile, Comisión Económica para América Latina y el Caribe (CEPAL), 112 pp.

Fernández, J. y Nieves, A. (1999). La cooperación entre empresas, análisis y diseño, España, ESIC.

Freeman, L. (1978). "Clarificación Conceptual”. Social Networks, Traducción de Herrero Reyes, pp. 215-239.

Fujita, M. et al. (2000). Economía espacial. Las ciudades, las regiones y el comercio internacional, traducción de Aurora Alcatraz, España, Ariel, 363 pp. (Título original: The Spatial Economy).

González, B. (2005). “Modelo de red de cooperación en los parques tecnológicos: un estudio comparado". Papeles de trabajo del Instituto de Estudios Fiscales, P. T. N. $17 / 06$.

González, T. (2007). “Las redes de cooperación empresarial internacionales vs redes locales" en Revista Venezolana de Gerencia, enero-marzo de 2007, Venezuela.

González, T. (2004). “Topología de las redes de cooperación internacionales: una propuesta", en la Memoria del IX Foro, FCA, UNAM.

González, T. (2003). “Las redes de cooperación empresarial internacionales. Un camino hacia el mercado asiático para la PYME mexicana", en la Memoria del VIII Foro, FCA, UNAM. 
REDES- Revista hispana para el análisis de redes sociales

Vol. 14,\#10, Junio 2008

http: // revista-redes.rediris.es

Grabher, G e lbert, O (2005). "Bad company? The ambiguity of personal knowledge networks". Journal of Economic Geography, Oxford University, N. pp. $1-21$.

Granovetter, M. (1973). The strength of weak ties. American J ournal of Sociology, vol. 78, n.6, pp. 1360-1380.

Kilkenny, M y Nalbarte, L. (2003). "Keystone Sector Identification. A Graph TheorySocial Network Analysis Approach". Regional Research Institute, West Virginia University, 1-32 pp.

Kilkenny, M. (2005). Community Networks: Cross Section Statistics and Analysis. Paper presented at the $19^{\text {th }}$ Pacific Regional Science Conference (PRSCO), July 2528, Nihon University, Tokyo, Japan, pp. 1-21.

Koschatzky, (2002). "Fundamentos de la economía de redes. Especial enfoque a la innovación". Economía industrial, n. 346. pp. 15-26.

Krugman, P. (1996). La organización espontánea de la economía, traducción de Mónica Martín, España, Antoni Bosch, 168 pp. (Título original: The Self-Organizing Economy).

LAX y Sebenius, (1991). El directivo como negociador, Madrid, Instituto de Estudios Fiscales, Ministerio de Economía y Hacienda [s.p.].

Le Bars, et al. (1998). "Innovation in SMEs: The Missing Link". High-Technology Small Firms Conference, Vol. 1 pp. 307-324.

Lippman, S. y Rumelt, R. (1982). “Uncertain Imitability: An Analysis of Interfirm Differences in Efficiency under Competition". Bell J ournal of Economics, vol. 13, pp. 418-438.

Maskell, et al. (2006). "Building Global Knowledge Pipelines: The Role of Temporary Clusters". European Planning Studies, Vol. 14, N.8, pp. 997-1013.

Pérez, J. et. al (2004). Teoría de Juegos, España, Pearson educación, 528 pp.

Petras y Veltmeyer (2004). Las privatizaciones y la desnacionalización de América Latina, Buenos Aires, Prometeo, 240 pp.

Porter, M (1998). Competitive Strategy: Techniques for Analyzing Industries and Competitors, $432 \mathrm{pp}$.

Powell, W. (1990). "Neither market nor hierarchy. Networks forms of organization". Cummings.

Rumelt, R. (1984). "Toward a Strategic Theory of the Firm". Competitive Strategic Management. Lamb (ed), Prentice-Hall. Englewood Cliffs, New Jersey, pp. 556-570.

Sáez, D. y Cabanelas, J. (1997). Cooperar para competir con éxito, España, Pirámide, $148 \mathrm{pp}$.

Sarason, S. et al. (1978). Resources, Community and Exchange Networks, San Francisco: Jossey Bass. 
REDES- Revista hispana para el análisis de redes sociales

Vol.14,\#10, Junio 2008

http://revista-redes.rediris.es

Schmitz, H. (1999). "Global competition and local co-operation: success and failures in the Sinos Valley, Brasil". World Development Studies, N. 27, pp. 16271650.

Suárez, C. (2006). "Situación sociodemográfica de los pueblos indígenas de México" en Pueblos indígenas y afrodescendientes de América Latina y el Caribe: información sociodemográfica para políticas y programas, Santiago de Chile, Naciones Unidas, 596 pp.

Torras, J. (1995). "Redes comerciales y auge textil en la España del siglo XVIII" en Maxine Berg, Mercados y manufacturas en Europa, traducción de Jordi Beltrán, Barcelona, Crítica, 256 pp. (Título original: Markets and Manufacture in Early Industrial Europe).

Vázquez, A. (1999). Desarrollo, redes e innovación, lecciones sobre desarrollo endógeno, España, Pirámide, 268 pp.

Wasserman, S. y Faust, K. (1994). Social Network Analysis: methods and applications. Nueva York: Cambridge University Press.

Waxell, A. y Malmberg, A. (2007). "What is global and what is local in Knowledgecreating interaction? The case of the biotech cluster in Uppsala, Sweden". Entrepreneurship and Regional Development, 19, pp. 137-159.

Williamson, O. y Winter W. (1996). La naturaleza de la empresa. Orígenes, evolución y desarrollo, Nueva York, 1991, traducción de Eduardo Suárez, México, Fondo de Cultura Económica, 326 pp.

Yin, R. (2003). Case study research. Design and Methods, Sage Publications, tercera edición, $178 \mathrm{pp}$. 